\title{
ANALYSIS AND SYNTHESIS OF ENHANCED BEE COLONY OPTIMIZATION WITH THE TRADITIONAL BEE COLONY OPTIMIZATION TO SOLVE TRAVELLING SALES PERSON PROBLEM
}

\author{
Prateek Agrawal \\ Lovely Professional \\ University, India
}

\author{
Harjeet Kaur \\ Lovely Professional \\ University,India
}

\author{
Deepa Bhardwaj \\ M.Tech (CSE) student \\ Lovely Professional \\ University, India
}

\begin{abstract}
Bee colony optimization is the recent swarm intelligence technique which has been applied to solve many combinatorial problems. In this paper we propose the enhanced bee algorithm based on Kmeans clustering to solve TSP. In the proposed algorithm parallel bee algorithm has been applied to each cluster and connection method has been suggested to combine the sub tour to global tour of the whole cities. It was found the enhanced bee algorithm give the better result and more optimal tour.
\end{abstract}

\section{Keywords}

Travelling Salesperson problem, Combinatorial Optimization, Bee Colony Algorithm, K-Means Clustering

\section{INTRODUCTION}

Swarm based metaheuristic has become motivation to many research scientists of related fields in recent years. Bonabeau has defined the swarm intelligence as "any attempt to design algorithms or distributed problem-solving devices inspired by the collective behaviour of social insect colonies and other animal societies" [1]. Swarm based optimization algorithm mimic the social behaviour of species. Bee algorithm is inspired by the food foraging behaviour of honey bees and could be regarded as belonging to the category of "intelligent" optimization tools [2].Bee algorithm have been developed to reach the near-optimum solution to large optimization problem. Traveling Salesman Problem (TSP) refers to a salesman who wants to find the cheapest way so that it visits every city exactly once and returns back to the starting city. When he takes his tour he must visit every point once. The cost of tour directly proportional to the tour length. Given a list of $\mathrm{N}$ cities

$$
\begin{aligned}
& \mathrm{V}=\left\{\mathrm{v}_{1}, \mathrm{v}_{2}, \ldots, \mathrm{v}_{\mathrm{N}}\right\} \text { and distances between them } \\
& \mathrm{d}=\left\{\mathrm{d}_{11}, \mathrm{~d}_{12}, \ldots, \mathrm{d}_{1 \mathrm{~N}}, \ldots, \mathrm{d}_{\mathrm{NN}}\right\}
\end{aligned}
$$

Where $d_{i j}$ gives the distance from node $v_{i}$ to $v_{j}$. The TSP aims to find a shortest tour path that visits each city only once and comes back to initial city. If $\mathrm{d}_{\mathrm{ij}}=\mathrm{d}_{\mathrm{ji}}$ then it is symmetric travelling salesman problem (STSP) otherwise it is asymmetric travelling salesman problem (ATSP)

The traveling salesman problem (TSP) [3] is a well-known nondeterministic polynomial-time (NP)-hard problem in combinatorial optimization. Travelling Salesman Problem has been solved by Nearest Neighbour hood, Greedy Heuristics [4], Christofides Heuristics[5],Simulated Annealing[6],Tabu Search[7].However, these methods are not always efficient for large scale problems. Modem heuristic approaches can get near optimal solutions in a reasonable time. Many metaheuristic algorithms have been applied to this problem for obtaining better results in acceptable computational times. Some of these algorithms employing metaheuristic approaches such as Genetic Algorithm (GA)[8], Ant Colony Optimization (ACO)[9] , Particle Swarm Optimization (PSO) [10] or Bee Colony Optimization (BCO) [11] were applied to solve TSP.

\section{REVIEW OF EXISTING WORK}

Chin Soon Chong[12 ] introduces the a bee colony optimization algorithm to job shop scheduling the experimental results show Bee algorithm achieves better mean and maximum percentages as well as higher number of best solutions. Li-Pie Wong[11] presented solution for travelling salesman problem using bee colony optimization involving evaluation of probability using arc fitness and the distance between the cities $i$ and $j$ respectively as the parameters. Waggle dance is used as the communication tool among bees. The dance duration of the bee is evaluated on the scaling factor, profitability score of a bee and average profitability bee colony. Li-Pie Wong [15] presented solution to the travelling salesman problem using the 2opt heuristic to further improve the solution.2opt heuristic bee algorithm achieves better results by evaluation of probability using arc fitness and the distance between the cities $i$ and $j$ respectively as the parameters. Li-Pie Wong [17] proposed Bee Colony Optimization Algorithm for Traveling Salesman Problem using Frequency based pruning strategy (FBPS) and fixed-radius near neighbour (FRNN) 2-opt. D.T.Pham [13] describes the application of Bee Colony algorithm for combinatorial optimization and functional optimization.

\section{BEE COLONY OPTIMIZATION}

\subsection{Background of Bee Algorithm}

Bee algorithm is a swarm intelligence technique which is based on foraging behavior of honey bees. The foraging behavior in a bee colony remains mysterious for many years until von Frisch translated the language embedded in bee waggle dances [14]. The natural bees have very good capability for searching food sources. The way of communicating information among the bees is the most important part of their foraging behaviour. Waggle dance is used by bees to convey information to other bees about the direction, distance and the quality of food. Upon finding the food source food it begins to dance in a figure eight pattern. After performing waggle dance on the dance floor, follower bees that were waiting inside the hive follow the dancer bee. The number of follower bees assigned to a patch is directly proportional to the quality of the patch. With this process the follower bee find food quickly and efficiently. Waggle dance is performed again when bees return to the hive. More bees will be recruited to that source if the patch is still good enough as a food source. Bees will 
stop collecting the poor quality of food and adjust strategy of searching food based on shared information to find the good quality food. Bee algorithm

Scout bee

1) Scout bee moves randomly from the hive in search of food source.

2) The bees keep on exploring the food sources until they are back to hive or out of energy.

3) Upon returning to the hive the scout bee shares its knowledge about exploration of food source by performing the waggle dance. Waggle dance is figure eight pattern dance which give information about distance, direction and quality of food source.

4) The scout bee performs the path construction.

Forage Bee

1) These are the bees which observe the dance performed by the scout bee, and get information about the food source direction and distance.

2) The forage bee goes to the food source on the basis of information it gets from the waggle dance

3) The forage bees perform the path reconstruction.

There are many types of bees which play role in bee algorithm which are classified under worker bee. Worker bees are thousands in number. They can be differentiated by their behavior.

Path construction:

In this step scout bee explore the entire food source that is number of cities available. The exploration process for the scout bee start from the $i^{\text {th }}$ city, they continue the exploration until the stopping criteria is met. Scout Bee performs the waggle dance on the dance floor to other hive mate once it has computed the whole tour. When bee performs the dance, it is performed for certain duration which depends on the quality of food. When the forage bee select the dance, it will use the path taken by the scout bee performing the dance as a guidance for exploring the food source known as preferred path. $P f_{i}$ denote the profitability rating for a bee ${ }_{i}[12]$.It is represented by:

$P f_{i}=\frac{1}{L_{i}}$

Where $L_{i}=$ the tour generated by bee $e_{\text {. }}$ The bee colony's average profitability rating, $P f_{\text {colony }}$ is given by

$$
P f_{\text {colony }}=\frac{1}{n} \sum_{i=1}^{n} P f_{i}=\frac{1}{n} \sum_{i=1}^{n} \frac{1}{L}
$$

Where $n=$ number of bees performing the dance. The dance duration is given by $D_{i}$, where $i$ represent any bee.

$D_{i}=K \cdot \frac{P f_{i}}{P f_{\text {colony }}}$

The probability $r_{i}$ of following a path is adjusted according the profitability ratings of a forager and the colony based on the lookup table 1.

Table 1: Probability Rating

\begin{tabular}{|l|l|}
\hline Profitability Rating & $\boldsymbol{R}_{\boldsymbol{i}}$ \\
\hline Pfi $<0.9$ Pfcolony & 0.60 \\
\hline 0.9 Pfcolony $<$ Pfi $<0.95$ Pfcolony & 0.20 \\
\hline 0.95 Pfcolony $<$ Pfi $<1.15$ Pfcolony & 0.02 \\
\hline 1.15 Pfcolony $<$ Pfi & 0.00 \\
\hline
\end{tabular}

Path Restructuring:

In this step the forage bees exploits the path selected by the scout bees.[11] The forage bees construct the solution for the TSP. Starting from the initial city the forage bee traverse every city in the graph only once, and comes back to initial city. When the forage be is at city $y_{i}$, it can only moves to next city that are defined in the allowed cities and giving precedence to city in preferred path. Bee uses the transition rule for choosing the next visiting city. The probability is denoted by $\mathrm{P}_{\mathrm{ij}}(\mathrm{t})$, which measure the possibility to move from city $y_{i}$ to city $_{j}$ at time t. The probability is defined by the equation given below. Probability is calculated in term of the distance and the fitness of arc.

$P_{i j}(t)=\frac{\left[\left[\rho_{i j}(t)\right]^{\alpha} \cdot\left[\frac{1}{d_{i j}}\right]^{\beta}\right]}{\sum_{j \in A_{i}(t)}\left[\rho_{i j}(t)\right]^{\alpha} \cdot\left[\frac{1}{d_{i j}}\right]^{\beta}}$

$\rho_{i j}(\mathrm{t})$ is defined as the fitness of arc from city $\mathrm{i}_{\mathrm{i}}$ to city $\mathrm{y}_{\mathrm{j}}$ at time $\mathrm{t}$ and $d_{i j}$ is the distance between the city $y_{i}$ and city. This concludes shorter the distance more chance to visit city. $\alpha$ is the variable for the influence of arc of fitness and $\beta$ is the variable for the influence of heuristic distance.

$\rho_{i j}=$ arc of fitness between city and $_{\text {city }}$ based on the preferred path

$d_{i j}=$ heuristic distance between city $_{i}$ and city

$P_{i j}=$ probability to going from city and $_{i}$ city $y_{j}$

$A_{i}=$ Allowed cities from city

The $\rho_{i j}$ arc of fitness between city $y_{i}$ and $c$ ity $y_{j}$ based on the preferred path is given by

$\rho_{i j}(t)= \begin{cases}\lambda & , j \in F_{\mathrm{i}}(\mathrm{t}) \\ \frac{1-\lambda \mid A_{i}(t) \cap F_{i}(t) \|}{\mid A_{i}(t)\|-\| A_{i}(t) \cap F_{i}(t) \|} & , j \notin F_{i}(t)\end{cases}$

$\lambda=$ value assigned to the preferred path, $\lambda<1.0, A_{i}(t)=$ number of allowed nodes, $\left|A_{i}(t) \cap F_{i}(t)\right|=$ number of preferred path, i.e 1 or 0

Path Selection:

This is the final step of the algorithm performed by forage bee, it select minimum total path calculated. 


\section{PROPOSED WORK}

\subsection{K-Means Clustering}

Kmeans algorithm is used for the clustering. The Kmeans clustering provides the better possibility of modeling and managing the overlapping of clusters. Kmeans clustering algorithm is used to classify or to group object on basis of attribute and feature into $\mathrm{k}$ number of groups where $\mathrm{k}$ is a positive integer. Define $\mathrm{k}$ centroid for each cluster. The placement of centroid must be in cunning way because different location causes different results. In the first phase of unsupervised Kmeans algorithm the $\mathrm{K}$ points are placed into the space represented by the objects that are being clustered. These points represent initial group centroids and assign each object to the group that has the closest centroid. In the second phase after assigning objects we recalculate the position of the $\mathrm{k}$ centroids. Repeat till the centroids no longer move. This put the objects into groups from which the metric to be minimized can be calculated.

\subsection{Methodology}

In our approach we are classifying the cities based on the clustering algorithm. Clustering of cities helps use to reduce the number of calculations and mathematical complexity. After clustering we are applying the parallel bee colony algorithm to calculate the sub tours of tsp in each cluster. We are proposing the connection method to combine the sub tour to global tour of the whole cities.

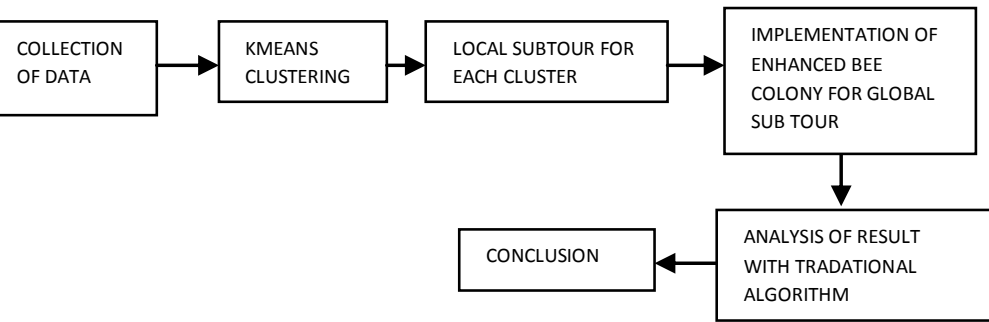

Fig1: Block Diagram of proposed methodology

\subsection{Proposed Algorithm}

(1) Apply kmeans clustering

(2) For each cluster do

2.1 Initialize the Population.

2.2 while stop criteria are not fulfilled do

2.3 while all bees have not built a complete path do

2.4 Observe Dance( )//Choose the dance with minimum duration

2.5 Forage ByTransRule( ) //Choose the next path depending on probability

2.6 Perform Waggle Dance ( )

2.7 end while

2.8 end while

2.9 Store the result with optimal value for each cluster

(3) Global tour is computed by starting from randomly choosing the cluster

(4) For cluster number 1 to $p-1 / / p$ is the maximum number of cluster

4.1 Observe_dance( )// stored optimal sequence is chosen

4.2 Forage_ByTransRule( )

4.3 From last city of each cluster move to next cluster by choosing the minimum of all allowed cities

(5) For $\mathrm{p}^{\text {th }}$ cluster reserve the node from list of allowed city which has minimum distance to initial city

5.1 Observe dance( )// stored optimal sequence is chosen

5.2 Forage ByTransRule( )

(6) traverse reserved node

(7) visit initial city

(8) store the optimal solution

\section{EXPERIMENTAL RESULTS}

The performance of proposed bee algorithm has been analyzed by applying them of the benchmark problem taken from TSP library. The numbers of bee equal to number of cities. The parameter setting used in the experiment are $\alpha=1, \beta=10$, $\lambda=.096, \mathrm{~K}=0.1 * \mathrm{BC} \max , \mathrm{BCmax}=10000$. The two performance parameter used in this paper are best, average path length. The proposed algorithm results are compared with the traditional bee colony optimization and the Bee System.

The assumption that are used in the experiment are

1) Bee hive is equidistant to all the cities

2) Each city is only visited once and be comes back to initial city

3) Decision for choosing the next city depends on arc of fitness and the distance between the cities

4) Each forage bee exploits the path of scout bee.

5) Bee explore the food source till the stopping criteria or the all the cities are traversed.

\section{CONCLUSION}

In the proposed enhanced bee algorithm based on Kmeans clustering to solve TSP, results shows that enhance bee colony optimization has reduced cost and enhanced performance for solving TSP problem. In future $\mathrm{K}$ means clustering can be further enhanced to get more optimal results. We can use different hard clustering algorithm to solve the problem. .

\section{ACKNOWLEDGMENTS}

We would like to express our greatest gratitude to the people who have helped \& supported us throughout this paper. We are grateful to our teachers, and my friends M.Sunil Babu and Pallavi Arora for their continuous support for the paper, from initial advice \& contacts in the early stages of conceptual inception $\&$ through on going advice $\&$ encouragement to this day. 
Table2 : Table showing the comparison of TSP path length

\begin{tabular}{|l|l|l|l|l|l|l|}
\hline \multirow{2}{*}{$\begin{array}{l}\text { Problem } \\
\text { Instances }\end{array}$} & Optimal & \multicolumn{2}{|l|}{ Bee Colony Optimization[11] } & Bee System[14] & Proposed Algorithm \\
& & \multicolumn{2}{|c|}{ Best Average } & & Best \\
\hline EIL51 & 426 & 428 & 429.62 & 431.121 & 426 & 427.32 \\
\hline EIL76 & 538 & 539 & 548.81 & n/a & 538 & 540.73 \\
\hline KROA100 & 21282 & 21762 & 22011.97 & 21441 & 21282 & 21415 \\
\hline
\end{tabular}

\section{REFERENCES}

[1] E. Bonabeau, M. Dorigo, G. Theraulaz, "Swarm Intelligence: From Natural to Artificial Systems", New York, NY: Oxford University Press, 1999

[2] D.T. Pham, A. Ghanbarzadeh, E. Koc, S. Otri, S. Rahim, M. Zaidi. "The Bees Algorithm - A Novel Tool for Complex Optimisation Problems" Proceedings of 2nd Virtual International Conference on Intelligent Production Machines and Systems (IPROMS 2006), The Internet, July 2006.

[3] D.L.Applegate, R.E.Bixby,V.Chvatal, and W. J. Cook, "The Traveling Salesman Problem: A Computational Study", Princeton University Press, 2006

[4] D.J Rosenkrantz,R.E Stearns and P.M Lewis" An Analysis of several heuristics for the travelling sales man problem" SIAM journal on computing, vol 6 ,no. 3,pp.563-581,1977

[5] A.M Frieze,"An extension of theChristofides Heuristics to the k-person travelling sales person problem," discrete applied mathematics, vol 6,n0 1,pp 79-83,1983

[6] J.W. Pepper, B.L. Golden and E.A. Wasil, "Solving the traveling salesman problem with annealing-based heuristics: a computational study," IEEE Transactions on Systems, Man and Cybernetics-Part A, vol.32, pp.72-77, 2002.

[7] Y. He, Y. Qiu, G. Liu and K. Lei, "A parallel adaptive tabu search approach for traveling salesman problems," in Proceedings of IEEE International Conference on Natural Language Processing and Knowledge Engineering, 2005, pp.796-801.

[8] J.D. Wei and D.T. Lee, "A new approach to the traveling salesman problem using genetic algorithms with priority encoding," in Proceedings of Congress on Evolutionary Computation, 2004, pp.1457- 1464.
[9] Marco Dorigo "Ant Colonies for the Traveling Salesman Problem"IRIDIA,Université Libre de Bruxelles. IEEE Transactions on Evolutionary Computation, 1(1):5366. 1997

[10] W.L. Zhong, J. Zhang and W.N. Chen,“A novel discrete particle swarmoptimization to solve traveling salesman problem,"in Proceedings of IEEE Congress on Evolutionary Computation, 2007, pp.3283-3287.

[11] L. P. Wong, M. Y. H. Low, and C. S. Chong, "A bee colony optimization algorithm for traveling salesman problem," in Proceedings of Second Asia International Conference on Modelling \& Simulation (AMS 2008), 2008, pp. 818-823.

[12] C. S. Chong, Y. H. Malcolm Low, A. I. Sivakumar, and K. L. Gay, "A bee colony optimization algorithm to job shop scheduling," in Proceedings of the 2006 Winter Simulation Conference, 2006. pp. 1954-1961.

[13] D.T.Pham, A.Ghanbarzadeh, E.Koc, S.Otri, S.Rahim, M.Zaidi, "The Bees Algorithm - A Novel Tool for Complex Optimization Problems", International Virtual Conference on Intelligent Production Machine and Systems, 2006

[14] K. von Frisch, "Decoding the language of the bee," Science, vol. 185 , no. 4152, pp. 663- 668, 1974.

[15] L. P. Wong, M. Y. H. Low, and C. S. Chong”Bee colony optimization with local search for the Travelling sales person problem"

[16] Dusan Teodorovic, Panta Lucic, Goran Markovic, Mauro Dell' Orco "Bee Colony Electrical Optimization: Principles and Applications"8th Seminar on Neural Network Applications in Engineering, NEUREL-2006

[17] Li-Pei Wong,Malcolm Yoke Hean Low,Chin Soon Chong(2009)"An Efficient Bee Colony Optimization Algorithm for Traveling Salesman Problem using Frequency- based Pruning" 
ISSN: 2277-3061 (online)

Technology

April 2012
International Journal of Computers \&

Volume 2 No.2, 